\title{
ANOMALOUS TAG DIFFUSION IN THE ASYMMETRIC EXCLUSION MODEL WITH PARTICLES OF ARBITRARY SIZES
}

\author{
Anderson A. Ferreira \\ Departamento de Física, Universidade Federal de São Carlos, 13565-905, São Carlos, SP Brazil \\ Francisco C. Alcaraz \\ Universidade de São Paulo, Instituto de Física de São Carlos, C.P. 369,13560-590, São Carlos, SP, Brazil
}

\begin{abstract}
Anomalous behavior of correlation functions of tagged particles are studied in generalizations of the one dimensional asymmetric exclusion problem. In these generalized models the range of the hard-core interactions are changed and the restriction of relative ordering of the particles is partially brocken. The models probing these effects are those of biased diffusion of particles having size $S=$ $0,1,2, \ldots$, or an effective negative "size" $S=-1,-2, \ldots$, in units of lattice space. Our numerical simulations show that irrespective of the range of the hard-core potential, as long some relative ordering of particles are kept, we find suitable sliding-tag correlation functions whose fluctuations growth with time anomalously slow $\left(t^{\frac{1}{3}}\right)$, when compared with the normal diffusive behavior $\left(t^{\frac{1}{2}}\right)$. These results indicate that the critical behavior of these stochastic models are in the Kardar-ParisiZhang (KPZ) universality class. Moreover a previous Bethe-ansatz calculation of the dynamical critical exponent $z$, for size $S \geq 0$ particles is extended to the case $S<0$ and the KPZ result $z=\frac{3}{2}$ is predicted for all values of $S \in Z$.
\end{abstract}

PACS numbers: PACS number(s): 02.50.Ey, 05.70.Ln, 64.60.Ht, 05.40.+j

Dynamic and equilibrium properties of lattice gases, i. e., systems involving randomly moving particles with hard-core interactions, have received much attention within the last decades, mainly due to their intrinsic and nontrivial many body behavior. Several important results have been obtained for such systems of particles. In particular the study of tagged-particle diffusion in hardcore lattice gases with nearest-neighbor hopping revealed interesting nontrivial many body behavior 11-13]. In one dimension the rate of diffusion depends strongly if the diffusion is symmetric or not, i. e., has or not a prefered direction. In the symmetric case, the root-mean-squared displacement of a tagged particle increases as $t^{\frac{1}{4}}$ during a time interval $t$, behavior that is anomalously slow when compared with the asymmetric case where this quantity exhibits the standard diffusive behavior $t^{\frac{1}{2}}$. Majumdar and Barma 111 have shown that even in the asymmetric case it is possible to find anomalous behavior of special correlations of tag particles (sliding-tag correlations), where the difference of the tag coordinates of the particles changes in time with a fixed tag velovity $v_{\text {tag }}$. For arbitrary values of $v_{t a g}$, except for $v_{t a g}=v_{t a g}^{c}$, these correlations increase with a typical diffusive behavior $t^{\frac{1}{2}}$, while at the special value $v_{t a g}=v_{t a g}^{c}$, that depends on the density of particles, they increase anomalously as $t^{\frac{1}{3}}$. This last behavior can be understood by mapping the asymmetric diffusion model into the particle height interface model, whose fluctuations in the continuum limit are governed by the Kardar, Parisi and Zhang (KPZ) model [14].

*Electronic address: alcaraz@if.sc.usp.br
From the above results [1] it is clear that the relevant parameters ensuring anomalous behavior for correlations of tag particles are: the hard-core effect (that implies, due to the topology of a one dimensional lattice, a fixed relative ordering of particles), the right-left asymmetry of the diffusion, and the velocity in the tag space where the correlations are measured.

All these results are obtained by imposing that the tag particles has a unity size, in terms of lattice units, or equivalently the particles have a fixed relative order and two particles at the same site have an infinite hard-core repulsion. The aim of this paper is to obtain the anomalous behavior of these sliding-tag correlations in the case where we increase or decrease the range of exclusion of molecules as well we relax the constraint of relative ordering of the molecules. The variation of the exclusion effect will be done by considering the asymmetric diffusion of particles with arbitrary integer sizes $S=0,1,2, \ldots$, and the effect of ordering will be verifyed by considering a generalization of the model where two consecutive ordered particles, can violate partially their ordering. This last model, as we shall see, corresponds to a generalization to the case where the particles in the asymmetric diffusion have an effective negative "size" $S=-1,-2, \ldots$.

We consider initially a one dimensional periodic lattice of $N_{s}$ sites contaning $N_{p}$ particles of a fixed size $S$ $(S=0,1,2, \ldots)$. These particles occupy $S$ consecutive sites, having a hard-core repulsion of range $S$. In the case $S=0$ the range of infinite repulsion is zero and we may put an arbitray number of particles in a given site. The dynamics of the asymmetric diffusion is such that a particle is selected at random and with probability $p(q)$ the particle attempts to move by one unity of lattice spacing to the right (left). The movement is accepted if the hardexclusion allows the final configuration. The unit of time 
is given by $N_{p}$ attempts of motion. For a given number of particles $N_{p}$ the number of holes (positions where the particles are allowed to move) is given by $N_{s}-S N_{p}$ and the mean velocity $v_{p}$ of the particles is given by

$$
v_{p}=\frac{(p-q)\left(N_{s}-S N_{p}\right)}{N_{p}+N_{s}-S N_{p}}=(p-q)(1-\chi),
$$

where $\chi=N_{p} /\left(N_{s}-(S-1) N_{p}\right)$ plays the role of an effective density of particles in the system. A given configuration at time $\mathrm{t}$ is given by the lattice coordinates of the particles $\{y(n, t)\}$, where $n=1,2, \ldots, N_{p}$ are the sequential labels indexing the particles. Since independently of the size of molecules the asymmetric diffusion process does not change their order (even for the size $S=0$ particles), we may chose this ordering such that $y(n+1, t) \geq y(n, t)+S$, and the periodic boundary condition translate into $y\left(n \pm N_{p}, t\right)=y(n, t) \pm N_{s}$. A corresponding interface fluctuation model can be defined by considering $y(n, t)$ as the local height of the surface at the horizontal cordinate $n$. In this model, at each time step, with probability $p(q) y(n, t)$ increase (or decrease) by 1 , and the motion is performed if $y(n+1, t)-y(n, t)>S$ (or $y(n, t)-y(n-1, t)>S$ ). In the steady state, for $p>q$ (or $p<q$ ) the surface grows (or decreases) in the vertical direction, with velocity $v_{p}$.

In order to search for anomalous behavior of correlations we consider a set of correlations among the coordinates $\left\{y\left(n_{t}, t\right)\right\}$, of the tagged particles with label $\left\{n_{t}\right\}$ at time $t$ and the cordinate $y\left(n_{0}, 0\right)$ of a given particle $n_{0}$ at initial time $t=0$. The label difference of the tagged particles $n_{t}-n_{0}=v_{\text {tag }} t$ changes with time, and for convenience we write the tag velocity as $v_{t a g}=\rho v_{p} b$, where $\rho=N_{p} / N_{s}$ is the density of particles and $b=b(S, \chi)$ is a real parameter. The mean values of these coordinates

$$
\begin{aligned}
<y\left(n_{t}, t\right)-y\left(n_{0}, 0\right)> & =<y\left(n_{t}, 0\right)-y\left(n_{0}, 0>+v_{p} t\right. \\
& =\left(v_{p}+v_{t a g} / \rho\right) t=v_{F} t
\end{aligned}
$$

increases with the frame velocity $v_{F}=v_{p}(1+b)$. The sliding-tag correlation functions [1]-[13] we considered are given by

$$
\sigma\left(v_{\text {tag }}, t\right)^{2}=<\left[y\left(n_{t}, t\right)-y\left(n_{0}, 0\right)\right]^{2}>-v_{F}^{2} t^{2} .
$$

We have measured these correlations by extensive Monte Carlo simulations by varying the asymmetry parameters $p, q$, the density of particles $\rho$, and the size $S$ of the particles. We verified that irrespective of the size $S$ of the particles, as long the diffusion is biased $(p \neq q)$, anomalous behavior happens for special values of the tag velocity $v_{\text {tag }}$. The value of $v_{\text {tag }}$ depends on the density $\rho$, the asymmetry parameter $(p-q)$ and the size $S$ of the particles. Examples of these measurements, for the cases where $S=2$ and $S=0$ are shown respectively in Fig. 1 and 2, where the time dependence of the correlation (3) are shown for several values of the tag velocity. In Fig. 1 we consider $p=0.75, q=0.25$ and density $\rho=1 / 5$ in a lattice of $N_{s}=150000$ sites while in Fig. $2 p=0.75$, $q=0.25$ and $\rho=1 / 4$ in a lattice of $N_{s}=120000$ sites. The average slope of the curves on these figures shows that apart from crossover effects, due to the finite size of the lattice, we have a normal diffusive behavior for arbitrary values of $v_{t a g}$, except at $v_{t a g}=v_{t a g}^{c}=b_{c} \rho v_{p}$, where $b_{c}=0.4167$ for $S=2$ and $b_{c}=0.2$ for $S=0$. At those critical values the increase of the correlations is anomalously slow and our numerical results indicates $\sigma \sim t^{\nu}$ where $\nu=0.32 \pm 0.01$, for the $S=0$ particles and $\nu=0.33 \pm 0.01$ for the $S=2$ particles.

A constraint on the dependence of the parameter $b_{c}=b_{c}(S, \chi)$ can be derived by generalizing the arguments presented in 111 for unity size particles. Instead of describing the asymmetric diffusion in terms of the size $S$ particles we consider the diffusion of holes in the reverse order. This is possible since the holes does not change their relative order and behave as ordinary size $S=1$ particles traveling on an effective lattice with $N_{s}-N_{p}(S-1)=1-\chi(S-1)$ sites (allowed positions). The mean velocity of holes is $v_{h}=S \chi(q-p)$, where the factor $S$ appears because in their motion the holes jump particles with size $S$ [15]. We can consider an equivalent sliding-tag correlation function (3) for the holes, with the tag velocity $v_{t a g}^{h}=(1-S \rho) b^{h}(\chi) v_{h}$ and the frame velocity $v_{F}^{h}=\left(1-b^{h}(\chi)\right) v_{h}$. The correspondence between $b(S, \chi)$ and $b^{h}(\chi)$, describing the same fluctuations in both correlations (particles or holes) are obtained by imposing the equality [11] $v_{F}=v_{F}^{h}$. At the critical point hole and particle correlations should reveal the same anomalous behavior. Taking into account that the holes behave as ordinary $S=1$ particles, but traveling $S$ times faster in an effective lattice with $N_{s}-(S-1) N_{p}$ sites, we have by comparing the tag velocities, $\left(N_{s}-(S-1) N_{p}\right) S b_{c}^{h}(\chi)=N_{s} b_{c}(1,1-\chi)$. As a consequence we obtain the general relation

$$
\begin{aligned}
& \chi(1-\chi(1-S)) b_{c}(1,1-\chi)+(1-\chi) b_{c}(S, \chi)= \\
& 1+(S-1) \chi
\end{aligned}
$$

that recovers the known relation in the particular case where $S=1$ [11].

Our numerical experiments show that the critical values $b_{c}(S, \chi)$ do not depend on the asymmetry parameter as long the diffusion is asymmetric $(p \neq q)$. Moreover our results are consistent with the conjecture

$$
b_{c}(S, \chi)=\left(\frac{S}{1-\chi}+1-S\right) \chi
$$

The agreement of the above conjecture was tested exhaustively in numerical simulations for several values of $\rho, p, q$ and $S$. In Table I we present some of our estimates for the exponent $\nu$ at $p=0.75$ and using in the simulations the conjectured value $b_{c}(S, \chi)$ giving in (5). Our results are clearly consistent with the exponent $\nu=\frac{1}{3}$ for all values of $S$ and $\chi$.

Up to now our results indicate that for arbitrary range of exclusion ( $\operatorname{size} S=0,1,2, \ldots$ ) we can define appropri- 
ate sliding-tag correlation functions exhibiting anomalous behavior. The existence of such anomalous behavior is connected to the fact that the dynamical behavior of these exclusion models are in the KPZ universality class. In fact the Bethe-ansatz solution [16] of the quantum Hamiltonian associated to the asymmetric diffusion of particles with arbitrary size $S(0,1, \ldots)$ gives a dynamical critical exponent $z=\frac{3}{2}$ in the KPZ universality class.

A key ingredient in all the previous diffusion processes is that independently of the particle's sizes always their order are fixed (even for size 0 particles). Now we are going to consider more general models where this ordering requirement is partially brocken. These models will correspond to a generalization of the diffusion dynamics of size $S$ particles, for the situation where the particles have a negative "size" $S(S=-1,-2, \ldots)$. In those models the particles are ordered from left to right and occupy as before the positions $\left.\left\{y(n, t), n=1, \ldots, N_{p}\right\}\right)$, with the constraint $y(n+1, t) \geq y(n, t)+S$. Then for negative values of $S$ the $n$th particle not necessarily is on the right of the $(n-1)$ th particle, but can be at a distance $-S$ on the left of this particle. This negative size $S$ gives a measure of the ordering of the particles. In the limit $S \rightarrow-\infty$ we have no ordering at all and the particles should behave as noninteracting random walks, with the absense of any anomalous behavior. It is interesting to note that the related interface model, given by the surface with cartesian coordinates $\{n, y(n, t)\}$, can exhibt hills of arbitrary height but valleys of maximun depth $-S$, in units of lattice spacing.

In Fig. 3 we show some examples of our simulations for $S=-1, p=0.75, q=0.25$ and density $\rho=1$ in a lattice of $N_{s}=150000$ sites. As we can see in this figure an anomalous behavior is also detected at the critical value $b_{c}=0.1667$. This value indicate that the conjecture (5), that gives $b_{c}=\frac{1}{6}$ for the present case, is also valid for negative values of $S$. Similar results are also obtained for other negative values of $S$ (see Table I).

Inspired by those last results we were able to extend the Bethe-ansatz calculations, already known 16] for particles with size $S \geq 0$, for the equivalent Hamiltonian describing the particles with negative values of $S$ 17. In the extreme anisotropic limit $p=1, q=0$ the eigenenergies of the associated Hamiltonian is given by

$$
E=\sum_{j=1}^{N_{p}}\left(1-z_{j}\right) / 2
$$

where the roots $\left\{z_{j}\right\}$ are given by the Bethe-ansatz equations

$$
\left(1+z_{j}\right)^{N_{s}-S N_{p}}\left(1-z_{j}\right)^{N_{p}}=-2^{N_{s}} e^{-i \frac{2 \pi m}{N_{p}}} \prod_{l=1}^{N_{p}} \frac{z_{l}-1}{\left(z_{l}+1\right)^{S}},
$$

where $j=1, \ldots, N_{p}, m=0,1, \ldots, N_{p}-1$. The above equations are more difficult to handle analitically than the corresponding ones in the cases where $S \geq 0$, even

TABLE I: Some values of the estimates of the exponent $\nu$ of the correlation function (B) evaluated at $p=0.75$, and for molecules of size $S$. These results were obtained at the tag velocity $v_{t a g}^{c}=b_{c} \rho v_{p}$, with $b_{c}$ giving by (5)

\begin{tabular}{lcccc}
\hline \hline & $S=-2$ & $S=-1$ & $S=0$ & $S=2$ \\
\hline$\chi=\frac{1}{4}$ & 0.32 & 0.31 & 0.33 & 0.32 \\
$\chi=\frac{1}{5}$ & 0.31 & 0.32 & 0.31 & 0.33 \\
\hline \hline
\end{tabular}

TABLE II: Finite-size estimates $z_{N_{s}, N^{\prime}}$ of the critical dynamical exponent evaluated by solving (7) for $N_{s}=N_{p}$. The extrapolated results are also shown.

\begin{tabular}{cccccc}
\hline \hline$N_{s}, N_{s}^{\prime}$ & 50,52 & 100,102 & 150,152 & 200,202 & $\infty$ \\
\hline$S=-1$ & 1.61344 & 1.56046 & 1.54122 & 1.53097 & $1.500 \pm 0.001$ \\
$S=-2$ & 1.72222 & 1.62689 & 1.58882 & 1.56769 & $1.504 \pm 0.005$ \\
\hline \hline
\end{tabular}

at the "half-filling" $\chi=\frac{1}{2}$, where a simplification occurs 16, 18, 19. The real part of the energy gap $G_{N_{s}}=$ $E_{1}-E_{0}$ behaves for large $N_{s}$ as $\operatorname{Re}\left(G_{N_{S}}\right) \sim N_{s}^{-z}$, producing for a pair of lattice sizes $N_{S}, N_{S}^{\prime}$ the finite-size estimate $z_{N_{s}, N_{s}^{\prime}}=-\ln \operatorname{Re}\left(G_{N_{s}}\right) / \operatorname{Re}\left(G_{N_{s}^{\prime}}\right) / \ln \left(N_{s} / N_{s}^{\prime}\right)$ for the dynamical critical exponent $z$. We solved numerically (7) for $S=-1$ and $S=-2$ at the filling $N_{p}=N_{s}$. The estimates $z_{N_{s}, N_{s}+2}$ for some values of $N_{s}$ are presented in Table II. We see clearly that the exponent tend toward the $\mathrm{KPZ}$ value $z=\frac{3}{2}$.

In conclusion, our results indicate that as long some type of relative ordering exists $(|S|<\infty)$, always we are going to find anomalous behavior for the sliding-tag correlation (2) at the critical tag velocity $v_{t a g}=\rho v_{p} b_{c}$ obtained from (11) and (5). This fact can also be undertood in terms of the related interface growth model, and it implies that as long $|S|$ is finite, we should expect the long time fluctuations being governed by a KPZ model.

\section{Acknowledgments}

This work was support in part by Conselho Nacional de Desenvolvimento Científico e Tecnológico, CNPq, Brazil, and FAPESP, São Paulo, Brazil. 
[1] T. Harris, J. Appl. Probab. 2, 323 (1965).

[2] F. Spitzer, Adv. Math. 5, 246 (1970).

[3] R. Arratia, Z. Ann. Probab. 11, 362 (1983).

[4] P. M. Richards, Phys. Rev. B 16, 1393 (1977).

[5] P. A. Fedders, Phys. Rev. B 17, 40 (1978).

[6] S. Alexander and P. Pincus, Phys. Rev. B 18, 2011 (1978).

[7] H. van Beijeren, K. W. Kehr, and R. Kutner, Phys. Rev. B 28, 5711 (1983).

[8] T. Ligget, Interacting Particle Systems (Springer-Verlag, Berlin, 1985).

[9] A. Demasi and P.Ferrari, J. Stat. Phys. 38, 603 (1985).

[10] R. Kutner and H. van Beijeren, J. Stat. Phys. 39, 3317 (1985).

[11] S. N. Majumdar and M. Barma, Phys. Rev. B 44, 5306 (1991).

[12] M. Barma, J. Phys. A 25, L693 (1992).

[13] P.-M. Binder, M. Paczuski, and M. Barma, Phys. Rev. E 49, 1174 (1994).

[14] M. Kardar, G. Parisi, and Y.C. Zhang, Phys. Rev. Lett. 56, 889 (1986).

[15] In the case where the particles have size $S=0$ we have a hole at each lattice point, and consequently the mean hole velocity $v_{h}=0$, but the critical tag velocity is finite and by using the conjecture (1) is given by $v_{t a g}^{h}=\chi(q-$ $p)(1-\chi)$.

[16] F. C. Alcaraz and R. Z. Bariev, Phys. Rev. E 60, 79 (1999).

[17] The Bethe-ansatz equation we obtained are similar to the equation (59) of 16] with the replacements $N=N_{s}$,
$\tilde{S}=S, \epsilon_{+}=p, \epsilon_{-}=q$, and $r=n$.

[18] L. H. Gwa and H. Spohn, Phys. Rev. Lett. 68, 725 (1992), Phys. Rev. A 46, 844 (1992).

[19] D. Kim, Phys. Rev. E 52, 3512 (1995).

Figure Captions

Figure 1 - The time dependence of the sliding-tag correlations (3) with size $S=2$ particles for some values of $b$. The parameters of the Monte Carlo simulations are $N_{s}=150000, N_{p}=30000, p=0.75$. The averaged estimated slope for the curves are $1.00(b=1)$, $0.90(b=0.6), 0.84(b=0.3)$ and at $b=b_{c}$ we have the estimate $2 \nu=0.65 \pm 0.03$.

Figure 2 - Same as figure 1 for size $S=0$ particles. The parameters of the Monte Carlo simulations are $N_{s}=$ $120000, N_{p}=30000, p=0.75$. The averaged estimated slope for the curves are $0.97(b=1), 0.95(b=0.5)$, $0.93(b=0.4)$ and at $b=b_{c}$ we have the estimate $2 \nu=$ $0.63 \pm 0.03$.

Figure 3 - Same as figure 1 for size $S=-1$ particles. The parameters of the Monte Carlo simulations are $N_{s}=150000, N_{p}=150000, p=0.75$. The averaged estimated slope for the curves are $0.86(b=0.4)$, $0.81(b=0.3), 0.76(b=0.1)$ and at $b=b_{c}$ we have the estimate $2 \nu=0.63 \pm 0.03$. 


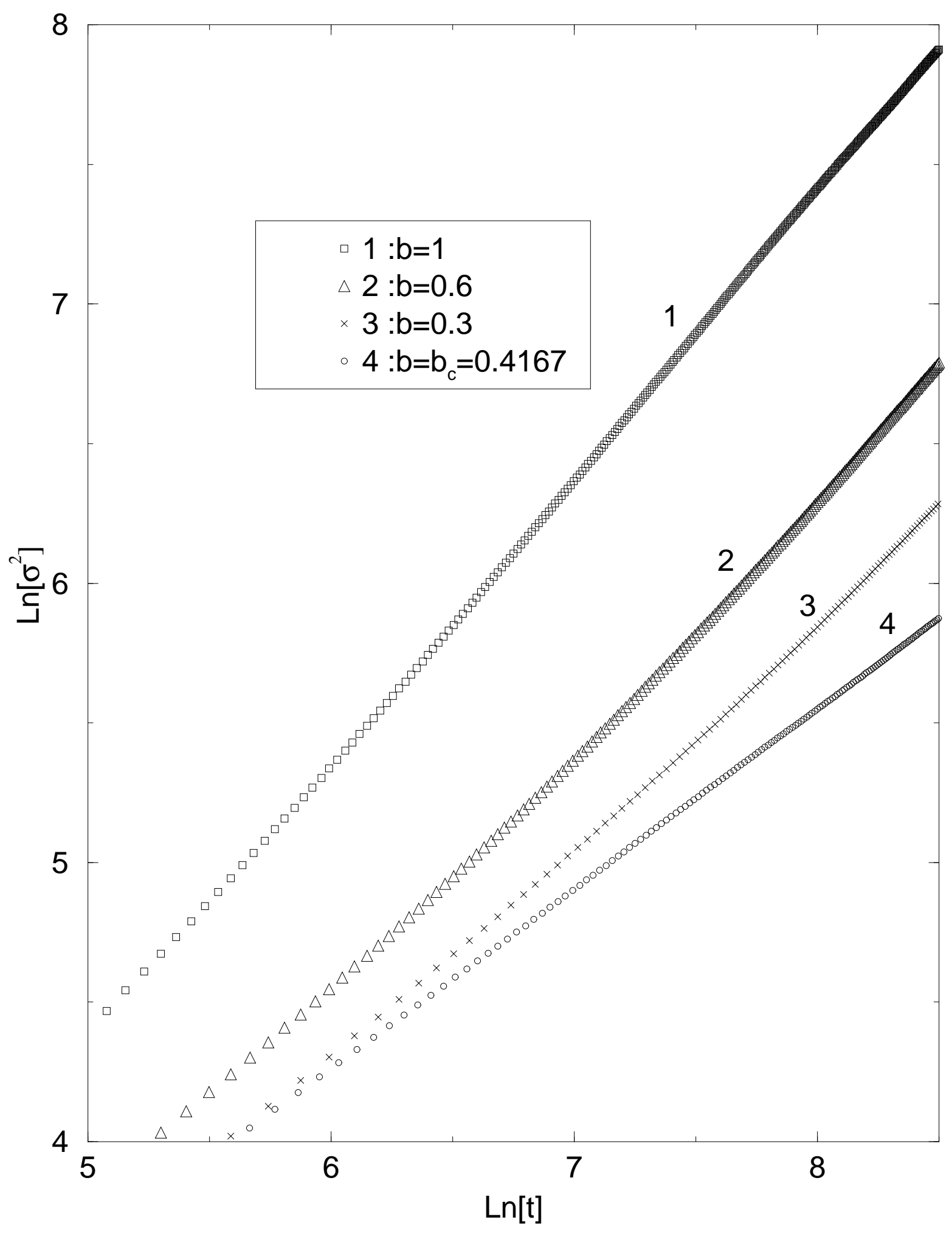




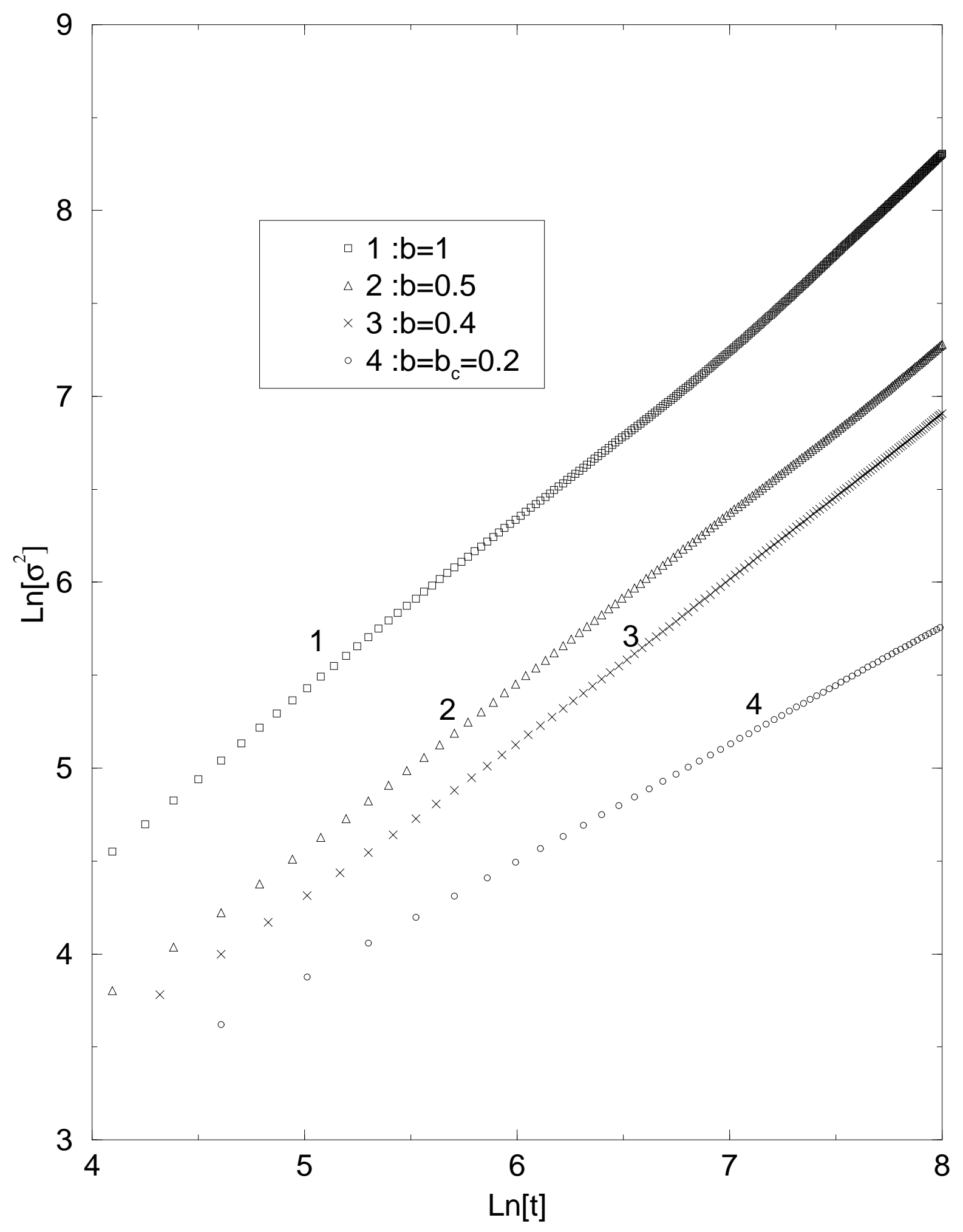




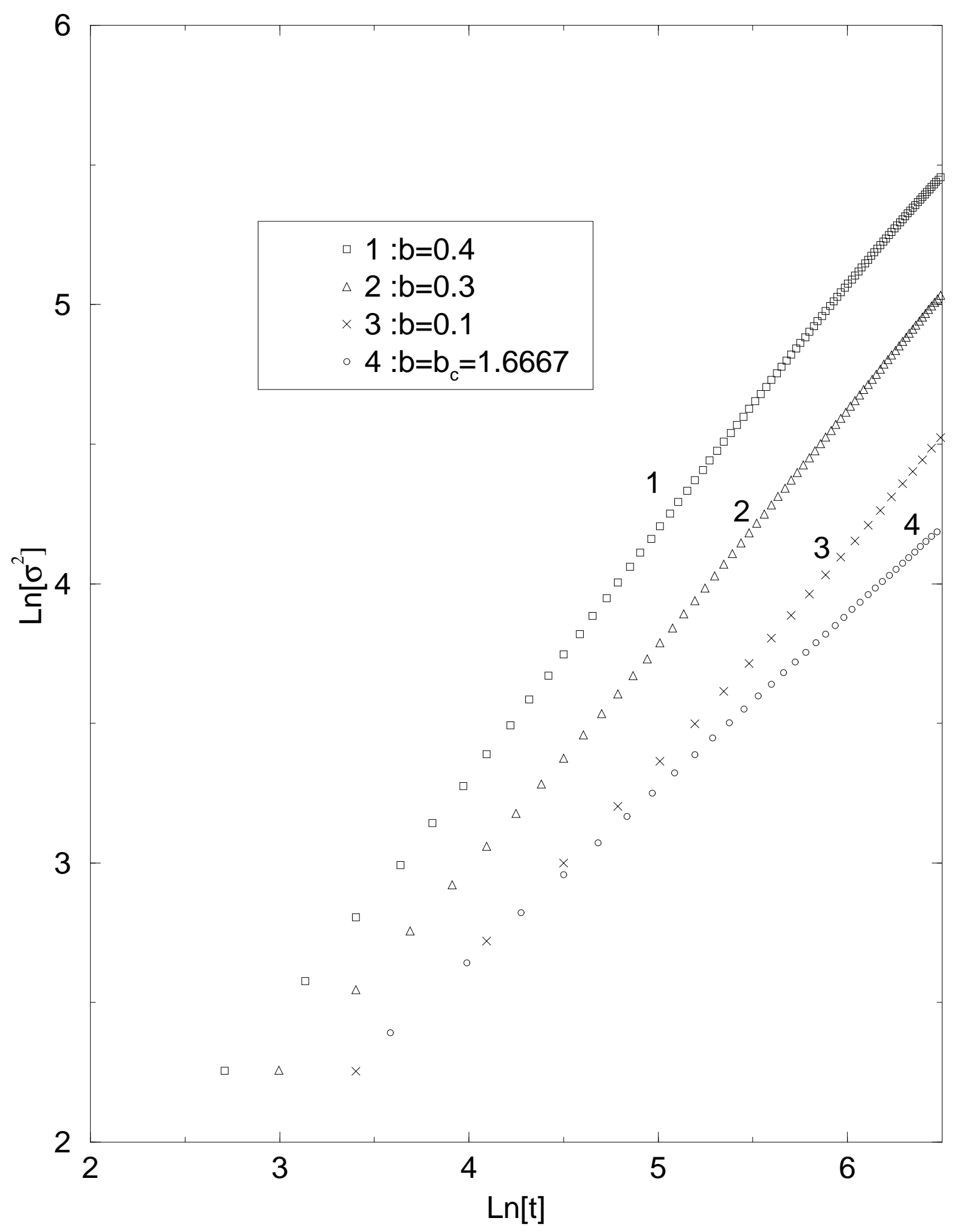

